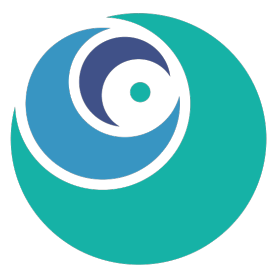

CIENCIA E INNOVACIÓNEN SALUD

\title{
Risk factors of acute confusional syndrome in elderly hospitalized in the Hospital Reina Sofía of Murcia. Retrospective case and controls
}

\section{Factores de riesgo del síndrome confusional agudo en ancianos hospitalizados en el Hospital Reina Sofía de Murcia. Estudio retrospectivo de casos y controles}

Irene Aragón ${ }^{1}$ Juan Rodado ${ }^{1}$

${ }^{1}$ Universidad de Murcia. Murcia, España.

Submitted 25 Jan 2018 Accepted 02 Feb 2018 Published 12 Abr 2018

\section{Editor in chief}

Isaac Kuzmar

editor@revcis.com

\section{Academic editor}

Juan Ávila

\section{Corresponding author}

Juan Rodado,

jvrodado9@hotmail.com

\section{DOI 10.17081/innosa.57}

(c) Copyright

2018 Rodado $^{1}$ \& Aragón. Distributed under Creative Commons CC-BY 4.0

\section{OPEN ACCESS}

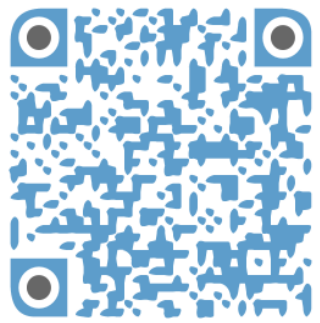

\section{ABSTRACT}

Background: Acute confusional syndrome is a current problem of special relevance among elderly patients admitted to hospital medical services. The determination of its risk factors is an essential process in the development and implementation of programs to prevent this complication

Methods: with the mentioned aim we have carried out this case-control study as an analytical, observational, retrospective and transversal study, whose source population was integrated by 60 patients over age 65 according to inclusion and exclusion criteria and divided into two groups: with and without delirium.

Discussion: Our analysis has confirmed the association between these factors and delirium: illness severity; previous history of Delirium (OR 10.6); mental status (OR 7.3); high risk medications (OR 6.9); renal failure (OR 6.5); medication at risk added (OR 6); physical status (OR 5.2); use of neuroleptics (OR 5.1); anemia (OR 4.75); sodium alterations (OR 4.5); urinary catheter (OR 3.8); low albumin (OR 3.7); infection (OR 3.1).

Conclusion: There is no relationship proved between acute confusional syndrom and the following factors: use of benzodiazepines, aggressive procedures, immobility, old age, dementia, diminished ADL skills, co-morbidity and polypharmacy, even if they have been identified as risk factors in previous studies. Hence, these results should be interpreted with caution.

\section{RESUMEN}

Introducción: El síndrome confusional agudo es un problema actual de especial relevancia entre los pacientes adultos mayores ingresados en los servicios médicos hospitalarios. La determinación de sus factores de riesgo es un proceso esencial en el desarrollo e implementación de programas de prevención de esta complicación

Métodos: Con el objetivo de identificar sus factores de riesgo, se ha llevado a cabo un estudio de casos y controles de tipo analítico, observacional, retrospectivo y transversal, cuya población a estudio ha estado constituida por 60 pacientes mayores de 65 años distribuidos en dos grupos: con y sin síndrome confusional.

Discusión: Los análisis pertinentes han permitido confirmar la asociación existente entre los siguientes factores y la aparición de SCA: severidad de la enfermedad; historia previa de SCA (OR 10,6); alteración del estado mental (OR 7,3); medicación crónica de alto riesgo (OR 6,9); insuficiencia renal (OR 6,5); medicación añadida de riesgo (OR 6); limitaciones del estado físico (OR 5,2); uso crónico de neurolépticos (OR 5,1); anemia (OR 4,75); alteraciones de los niveles de sodio en sangre (natremia) (OR $4,5)$; catéter urinario (OR 3,8); desnutrición (OR 3,7); infección (OR 3,1).

Conclusión: No se ha demostrado la relación del síndrome confusional agudo con los factores: uso crónico de benzodiacepinas, intervención agresiva, inmovilidad, edad avanzada, deterioro cognitivo, índice de comorbilidad, situación funcional previa y polifarmacia, pese a haber sido identificados como factores de riesgo en estudios previos y, por este motivo, se deberá ser cauteloso en la interpretación de los resultados obtenidos.

Keywords Acute confusional syndrome; delirium; risk factor; elderly inpatients Palabras clave Síndrome confusional agudo; delirium; factores de riesgo; ancianos 


\section{INTRODUCCIÓN}

La denominación de delirium apareció en el DSM-IV-TR como un intento de consolidar los múltiples términos existentes (síndrome confusional agudo, síndrome cerebral agudo, encefalopatía metabólica, psicosis tóxica, insuficiencia cerebral aguda, estado mental alterado, etc.) en una única etiqueta diagnóstica. De esta manera, los términos: delirium, confusión o síndrome confusional agudo (SCA) son tomados como sinónimos en la literatura. Sin embargo, el término ‘delirium' es el más utilizado.

Consiste en un síndrome de causa orgánica, en ocasiones plurietiológico, que se caracteriza por una alteración del nivel de conciencia y de la atención, así como de diversas funciones cognitivas, como la memoria, orientación, pensamiento, lenguaje o percepción. Tiene un comienzo agudo (en horas o días) y un curso fluctuante. Los pacientes con delirium tienen una alteración del nivel de atención, está disminuida su capacidad para centrar, mantener o dirigir la atención, además de la alteración de la percepción que puede hacer que el paciente malinterprete la realidad, tenga ilusiones o alucinaciones, pudiendo esto condicionar su comportamiento y expresar miedo o agresividad ante estímulos externos.

En mayo de 2013 fue publicada la quinta edición del Manual Diagnóstico y Estadístico de los Trastornos Mentales (DSM-5), lo que conllevó cambios en algunos criterios diagnósticos con respecto a la edición previa, entre ellos en los criterios de delirium. Según Sepulveda y Franco (2015) el DSM-5 en español desconcierta al denominar "síndrome confusional» al trastorno. Aunque esa denominación se relaciona con el concepto francés del siglo XIX, Confusion Mentale Primitive (que tuvo que ver con la consolidación del constructo actual del síndrome), la mayoría de los hispanoparlantes prefieren el vocablo delírium (Morandi, Pandharipande, Trabucchi, Rozzini, Mistraletti, Trompeo, 2008)

A pesar de ello en este trabajo utilizaremos el termino Sindrome Confusional Agudo ya que es el que aparece en el DSM-5, y son los criterios diagnósticos que hemos usado y que exponemos a continuación (American Psychiatric Association, 2013)

A. Una alteración de la atención (p. ej, capacidad reducida para dirigir, centrar, mantener o desviar la atención) y la conciencia (orientación reducida del entorno).

B. La alteración aparece en poco tiempo (habitualmente unas horas o pocos días), constituye un cambio respecto a la atención y conciencia iniciales y su gravedad tiende a fluctuar a lo largo del día.

C. Una alteración cognitiva adicional (p. ej. Déficit de la memoria, de orientación, de lenguaje, de la capacidad visoespacial o de la percepción).

D. Las alteraciones de los Criterios $\mathrm{A}$ y $\mathrm{C}$ no se explican mejor por otra alteración neurocognitiva preexistente, establecida o en curso, ni suceden en el contexto de un nivel de estimulación extremadamente reducido, como seria el coma.

E. En la anamnesis, la exploración física o los análisis clínicos se obtienen evidencias de que la alteración es una consecuencia fisiológica directa de otra afección medica, una intoxicación o una abstinencia por una sustancia (p. ej. Debida a un consumo de drogas o 
Por todo ello, el SCA debe se entendido como una enfermedad médica potencialmente tratable y, lo que es más importante, prevenible.

Existen varios motivos por los que parece necesario hacer un estudio acerca del síndrome confusional agudo; para empezar, la alta incidencia de delirium, especialmente en pacientes hospitalizados mayores de 65 años, donde las cifras oscilan entre el 10\% y el 40\% en la actualidad (Ganuza, González, Gaviria, 2012).

Además de la fuerte presencia de SCA en nuestro medio, otro de los factores que marcan su relevancia es que afecta de forma radical al pronóstico del paciente, puesto que se ha demostrado que desarrollar un síndrome confusional agudo se asocia a un mayor número de eventos negativos como pueden ser: mayor riesgo de complicaciones intrahospitalarias, mayor deterioro funcional con peor recuperación tras un proceso agudo, estancia hospitalaria más prolongada, institucionalización y aumento de la mortalidad tanto intra como extrahospitalaria en comparación con pacientes con problemas análogos pero en los que no se ha producido un SCA (I nouye, Rushing,Foreman, Palmer, Pom- pei, 1998; McCusker, Cole,Dendukuri, Belzile, Primeau, 2001; González, Martínez,Calderon, Villarroel, Yuri, Rojas, 2009; Curyto, Johnson,TenHave, Mossey, Knott, Katz, 2001)

A pesar de su frecuencia, de sus significativas consecuencias y de tratarse de un problema bien conocido y de gran relevancia clínica, el delirium es a menudo infradiagnosticado. No es detectado por el personal sanitario en cerca del $70 \%$ de los pacientes (Inouye, Foreman, Mion, Katz, Cooney 2001) especialmente en las formas de SCA que no presentan agitación psicomotriz y que por tanto pasan desapercibidas para el clínico y no van a beneficiarse del manejo sintomático del delirium que podría ayudar a controlar la gravedad de los síntomas.

El desafío actual frente al SCA necesita de la pronta identificación de sus factores de riesgo y la consecuente introducción de las medidas preventivas de las que disponemos en la actualidad, medidas preventivas claras y bien establecidas, de sencilla implementación en los servicios y que han demostrado en numerosas investigaciones ser eficaces en la disminución de la aparición del síndrome confusional agudo. Existe evidencia de que la implementación de programas de prevención de SCA logra reducir su incidencia; por ejemplo en el estudio de Gentric, Le Deun, Estivin (2007) en el que comparan la incidencia de síndrome confusional agudo antes y después de la puesta en marcha de una estrategia preventiva con medidas no farmacológicas, donde se observa que en el grupo sobre el que se ha hecho la intervención hay una reducción del riesgo relativo del $73 \%$.

Otro estudio realizado ha puesto de manifiesto el valor de estas medidas, incluso en términos económicos, con una reducción de costes superior al 15\% tan sólo midiendo la duración de las estancias de los ancianos (Leslie, Zhang,Bogardus, Holford, Leo-Summers, Inouye, 2005). Un buen conocimiento de los factores de riesgo del SCA, así como de sus mecanismos de producción más habituales, unido a una supervisión mantenida por parte del equipo multidisciplinar (conformado por: psiquiatra, médico internista, geriatra, enfermeros, etc.) constituyen las claves para llevar a cabo una prevención adecuada. 
En todo este contexto y sobre todo en los últimos años, ha nacido por parte de los clínicos la necesidad de implementar estrategias que permitan identificar los pacientes en mayor riesgo de delirium sobre los cuales se van a aplicar dichas medidas de prevención, y así se han dirigido los esfuerzos de las investigaciones en establecer una escala clínica predictiva: Inouye, Viscoli, Horwitz, Hurst, Tinetti (1993) desarrollaron un modelo predictivo de SCA incidente en pacientes mayores de 70 años, hospitalizados por patologías médicas, sin demencia ni delirium al ingreso. En la cohorte inicial se identificaron cuatro factores de riesgo: deterioro visual, enfermedad grave, deterioro cognitivo y elevación de la relación BUN/creatinina plasmática como marcador de deshidratación. Otro modelo de 1996 enfocado en los factores precipitantes incorpora cinco factores de riesgo independientes: uso de restricción física, uso de más de tres medicamentos, malnutrición, catéter urinario y eventos iatrogénicos (Inouye \& Charpentier, 1996). Otros modelos, como el de Pompei, emplean características demográficas, depresión, cognición, funcionalidad y alcoholismo (Pompei, Foreman, Rudberg, Inouye, Braund, Cassel, 1994) también en 1996 incluye demencia, severidad de la enfermedad y niveles de urea elevados (O’Keeffe \& Lavan, 1996).

Todos estos modelos citados hasta ahora no lograron, sin embargo, traspasarse a la práctica clínica habitual, posiblemente debido a que estas evaluaciones requieren personal entrenado y un tiempo no menor de aplicación, siendo ambos recursos escasos en el momento del ingreso. Más tarde, en 2014 el departamento de Medicina Interna de la Universidad Católica de Chile (Carrasco, Villarroel, Calderón, Martínez, Andrade, González, 2014) publica un nuevo modelo que a diferencia de los anteriores incorpora también aquellos pacientes que ya ingresan con delirium y que utiliza dos parámetros: edad y funcionalidad previa, esta segunda medida mediante el índice de Barthel con la limitación de que no evaluaron factores de riesgo bien conocidos como son la deprivación sensorial, uso de fármacos de riesgo, abuso de sustancias ni malnutrición.

El objetivo principal del trabajo es describir si existe asociación entre los factores de riesgo tradicionalmente relacionados con el SCA y la aparición de tal síndrome en pacientes ancianos hospitalizados en los distintos servicios del departamento de Medicina Interna del Hospital Universitario Reina Sofía de Murcia. Tenemos como objetivo secundario poder establecer en un segundo estudio un sistema de predicción clínico que pueda ser fácilmente aplicable y sirva como elemento para predecir la futura presencia del síndrome y así establecer las medidas preventivas y de control necesarias. La hipótesis esperada es que, en efecto, confirmemos la relación existente entre los factores de riesgo descritos en la literatura y la presencia de SCA en los pacientes de nuestro medio.

\section{MÉTODO}

\section{Diseño del estudio}

Este trabajo está planteado como un estudio de casos y controles, y por tanto, de tipo retrospectivo, observacional analítico.

Se ha recogido la muestra a partir de una población de pacientes hospitalizados en el Hospital Universitario Reina Sofía de Murcia durante los años 2014 y 2015 siguiendo unos criterios determinados de inclusión: la edad mayor de 65 años, puesto que es uno de los cuatro factores de riesgo incluidos en la guía de delirium NICE (Young, Murthy, Westby, Akunne, O’Mahony, 2010) , así como que los pacientes estuvieran hospitalizados un mínimo de una semana en servicios 
médicos.

Se ha considerado "caso" tanto a la muestra de la población cuyo motivo de ingreso fue un síndrome confusional agudo, como a aquellos que lo han desarrollado durante una hospitalización por otra causa médica. Han sido identificados a partir de las hojas de interconsulta realizadas a psiquiatría con sospecha de SCA, excluyendo aquellos cuyo diagnóstico final, basado en los criterios clínicos referidos con anterioridad, hubiera sido distinto de síndrome confusional agudo, así como los casos en los que éste síndrome hubiera estado relacionado con la suspensión o toma de alcohol $u$ otros tóxicos. Los pacientes que desarrollaron este trastorno pero no precisaron de interconsulta a un psiquiatra, porque su médico a cargo no lo vio necesario, no han sido contabilizados.

Se ha considerado "control" a la muestra de la población que aún habiendo estado en riesgo de desarrollar un delirium, no ha presentado un síndrome confusional agudo durante la hospitalización. Han sido identificados de manera aleatoria y sistematizada a partir de una lista de pacientes mayores de 65 años ingresados durante el año 2014 en los servicios médicos dependientes de medicina interna. Se han elegido tantos controles como el número de casos recogidos. El método de aleatorización usado para la elección de los controles ha sido el siguiente: se han seleccionado tres pacientes por cada mes de hospitalización, los tres primeros ingresos de cada día 15 de mes, dos ingresados a cargo de medicina interna y el tercero a cargo de otro servicio médico incluyendo cardiología, digestivo y neurología, con el fin de igualar esta proporción a la del grupo de casos previamente seleccionados. Se han excluido de este grupo tres pacientes que sí habían desarrollado un síndrome confusional agudo durante la hospitalización y de los cuales no existía interconsulta, añadiéndose al conjunto de "casos" seleccionados previamente.

La muestra final ha constado de 60 pacientes, distribuidos en: 30 con síndrome confusional agudo y 30 sin esta complicación.

Para el estudio de los factores de riesgo presentes en uno y otro grupo se ha realizado el análisis pormenorizado y la revisión de historias clínicas por medio del programa informático SELENE. La recogida de datos de ha realizado en los meses de marzo y abril de 2016.

\section{Análisis de los factores de riesgo. Variables y medidas.}

II. 1 Definición de factor de riesgo.

El término "riesgo" implica la presencia de una característica o factor (o de varios) que aumenta la probabilidad de consecuencias adversas. En este sentido el riesgo constituye una medida de probabilidad estadística de que en un futuro se produzca un acontecimiento por lo general no deseado.

El conocimiento y la información sobre los factores de riesgo tienen diversos objetivos, entre los que se encuentran: la predicción, la causalidad, el diagnóstico y la prevención.

II. 2 Análisis de los factores de riesgo de SCA.

En primer lugar, mediante la búsqueda sistematizada en la bibliografía, se han identificado y recogido la mayoría de los factores de riesgo de SCA que han sido estudiados, pretendiendo incorporar un rango lo más amplio posible. 
Se han podido diferenciar en factores de riesgo que podríamos considerar predisponentes, y aquellos factores que pueden identificarse como precipitantes o desencadenantes de síndrome confusional agudo.

Factores Predisponentes

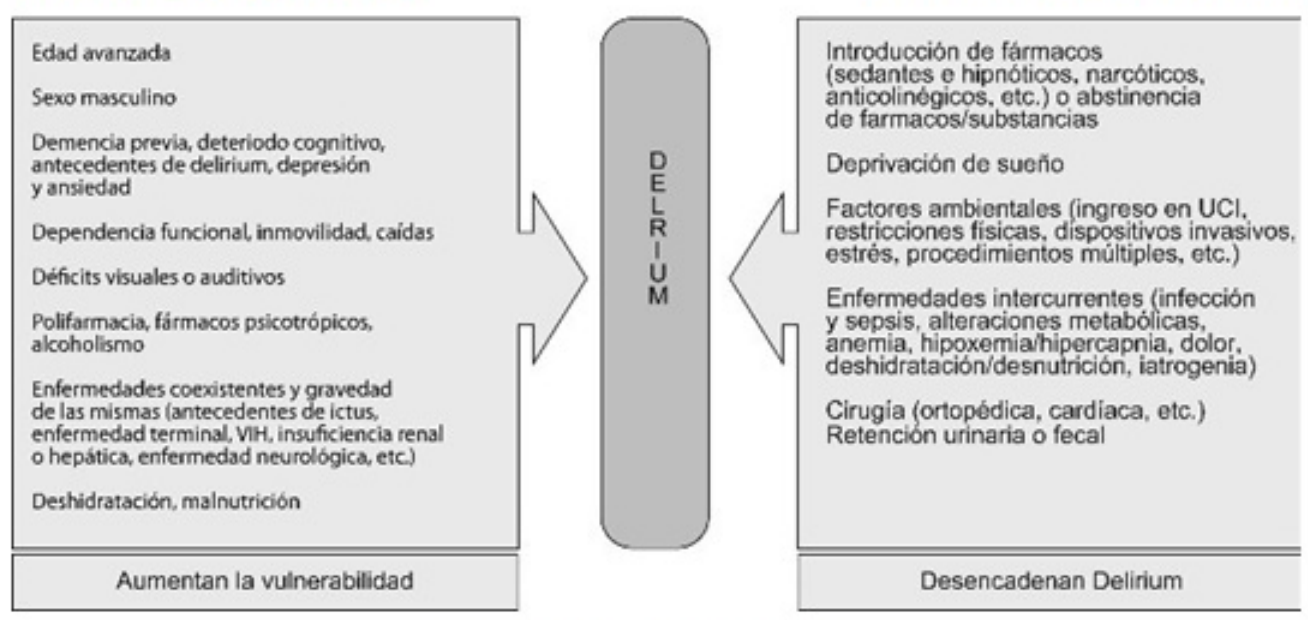

Fuente: Rex Esp Geriatr Gerontol. $2010^{18}$

II.3 Definición, medición y justificación de las variables estudiadas.

\section{-Factores demográficos}

Se han incluido tanto la (i) edad de cada paciente medida en años como si ésta era (ii) menor (0) o mayor de 75 años (1) así como el (iii) sexo femenino (0) o masculino (1).

La edad ha sido el factor de riesgo más frecuentemente estudiado según S. Ahmed y colaboradores (Ahmed, Leurent, Sampson, 2014) en una revisión sistemática y meta-análisis sobre los factores de riesgo del SCA incidente en 2014, que dejaba de manifiesto que a mayor edad existe un mayor riesgo de síndrome confusional agudo. En cuanto al sexo, concluyeron que no existía asociación significativa entre el sexo masculino y el SCA en los estudios que incluía la mencionada revisión.

\section{-Estado mental}

Dentro del estado mental, se han definido distintas variables como son la (i) depresión, (ii) demencia o deterioro cognitivo y (iii) otras alteraciones mentales (donde se incluirían otras enfermedades mentales/psiquiátricas distintas de las anteriores, como por ejemplo la esquizofrenia o los trastornos de la personalidad); determinando su ausencia (0) o presencia (1) según estuviera o no referido en los antecedentes personales activos de cada paciente o en el caso del deterioro cognitivo por un MMSE al ingreso con puntuaciones que así lo concluyeran.

Así mismo, se ha determinado una última variable dentro del estado mental, en la que (0) es la ausencia de los factores anteriores o la presencia de uno de ellos y el (1) significa que el paciente presenta dos o más de estos factores.

En el meta-análisis de S. Ahmed y Colaboradores19, ya citado anteriormente se observó que tanto la depresión como la demencia estaban significativamente asociadas con el delirium, ésta 
última en seis estudios (con una OR de 6.62), tres de los cuales eran análisis multivariantes.

\section{-Estado o enfermedad física}

En este apartado se han incluido tres variables:

Severidad de la enfermedad, medida mediante la escala APACHE II (escala avalada para pacientes de $\mathrm{UCl}$, que se ha intentado extrapolar a nuestros pacientes), que otorga valores entre (0-71) interpretándose que a mayor puntuación la enfermedad es más severa y hay un mayor riesgo de muerte. Cálculo realizado gracias a una herramienta facilitada por la sociedad de medicina intensiva de la comunidad de Madrid.

Carga de comorbilidad, medida mediante el índice de Charlson que predice la mortalidad a un año para un paciente que puede tener un abanico de condiciones comórbidas. Calculado a través de la plataforma de Internet de la sociedad andaluza de Medicina intensiva, Urgencias y Coronarias (SAMIUC).

Situación funcional previa, medida mediante el índice de Barthel que otorga valores entre (0-100) y se interpreta de la siguiente manera: 0-20: Dependencia total; 21-60: Dependencia severa; 61-90: Dependencia moderada; 91-99: Dependencia leve; 100: Independencia. Debido a la dificultad de rellenar la información para el adecuado cálculo de este índice, ya que nuestro estudio nos permite sólo un análisis retrospectivo, se ha medido de manera cualitativa mediante las referencias reflejadas en la historia clínica de cada paciente otorgándose el valor (0) tanto a la independencia como a la dependencia leve, y el valor (1) a la dependencia moderada o severa.

Como ya hemos venido citando, el meta-análisis de Ahmed, Leurent, Sampson (2014) concluye que en la mayoría de estudios incluidos en su revisión tanto la severidad de la enfermedad como la co-morbilidad son un factor de riesgo significativo en análisis tanto uni como multivariantes.

En cuanto a la situación funcional previa del paciente, además de en el meta-análisis ya indicado, donde se habla de "capacidades para las actividades de la vida diaria" y que confirma la asociación significativa, existen otros estudios, como el de Formiga et al. (2007), que demuestra que es un importante factor predisponente de SCA.

-Historia previa de síndrome confusional agudo

Para definir que no ha habido historia previa de SCA (0) o sí (1) se ha comprobado si este hecho estaba señalado de forma explícita en la historia clínica del paciente, en informes de altas anteriores al ingreso estudiado o en los antecedentes personales. Presenta como limitación que no se conocen los criterios diagnósticos utilizados para definir SCA en los informes previos al del episodio estudiado en la actualidad.

Aparece reiteradamente en la literatura como factor predisponente aunque no son muchos los estudios que analizan la asociación entre haber tenido previamente un síndrome confusional agudo y el riesgo de volver a desarrollarlo.

-Actividades de la vida diaria, visión y oído

Se han incluido dentro de esta categoría tanto (i) la deprivación sensorial (por motivos de disminución de la agudeza visual y/o ceguera, hipoacusia y /o sordera total) referida en las notas médicas o antecedentes personales de la historia clínica, (ii) la inmovilización o problemas de movilidad que limitaran al paciente a una vida de cama-sillón y (iii) la colocación de un catéter urinario durante el ingreso a estudio; variables, medidas como ausencia (0) o presencia (1) de 
alguno de estos impedimentos físicos. Por último, la cuarta variable dentro de esta categoría haría referencia a la limitación física general que presenta el paciente y sería interpretada como (0) si presenta uno o ninguno de los impedimentos anteriores, y (1) si presenta al menos dos de los factores especificados.

Ahmed, Leurent, Sampson (2014) en su revisión ya pusieron de manifiesto que las variables medidas dentro de esta categoría eran factores de riesgo de delirium estadísticamente significativos, con una mención especial para el cateterismo urinario, que se demuestra como factor de riesgo independiente en dos de los estudios más importantes que incluye el referido metaanálisis.

\section{-Patología intercurrente}

Esta es una de las categorías más amplias y donde se estudian la mayor parte de factores que son desencadenantes o precipitantes del episodio de delirium, dejando a un lado la medicación, y se trata de variables de gran importancia puesto que la mayoría de ellas pueden ser modificadas y por tanto, prevenibles; se citan a continuación:

- Infecciones. EI SCA es una forma frecuente de presentación atípica de la infección en ausencia de fiebre, leucocitosis o síntomas de localización y cuando aparece es obligada la búsqueda sistemática de signos de infección21. Las más frecuentemente relacionadas con delirium son la infección respiratoria y la urinaria. Para el análisis de esta variable se ha determinado la ausencia (0) o presencia (1) de infección de cualquier origen según estuviera así referido en las notas de evolución o el diagnóstico en el informe de alta del ingreso a estudio.

- Alteraciones del equilibrio hidroeléctrico. Se ha estudiado principalmente las alteraciones del sodio (tanto por hiper como por hiponatremia), usando como referencia el resultado del análisis de sangre realizado en urgencias o, en su ausencia, el primero de los análisis realizados durante el ingreso y midiéndolo como sin alteración (0) cuando el valor de la natremia se encontraba entre $136-145 \mathrm{mEq} / \mathrm{l}$, o con alteración (1) si se encontraba por encima o debajo de los mencionados valores. S. Ahmed y colaboradores 19 concluyó que investigaciones de laboratorio con sodio bajo o alto, eran muy frecuentemente asociadas con un riesgo aumentado de síndrome confusional agudo.

- Fiebre. Entendida como una temperatura corporal de 38ㄷ o más, medida como sí (1) o no (0) según estuviera referido en las hojas de evolución a lo largo del ingreso del paciente.

Enfermedades neurológicas primarias. Incluyendo dentro de estas el accidente cerebrovascular, la hemorragia intracraneal y los procesos infecciosos tales como meningitis o encefalitis. Siendo categorizadas según la ausencia (0) o presencia (1) de alguno de los referidos procesos neurológicos agudos.

- Dolor. El dolor de cualquier causa puede ser un factor precipitante que se debe tomar en consideración en todo paciente anciano. Para medir dicho factor en nuestro estudio, al ser una variable cualitativa de difícil determinación en un estudio retrospectivo, hemos considerado que había presencia de dolor (1) si se encontraba explícitamente referido en las notas de evolución y si dicho dolor había necesitado de la prescripción de analgésicos durante el ingreso.

- Insuficiencia de cualquier órgano o sistema. Se estudian tres variables, como son (i) el fracaso 
renal agudo, (ii) la insuficiencia respiratoria medida por la presencia o no de hipoxia según el paciente hubiera necesitado gafas nasales u otros mecanismos de ventilación durante el ingreso, y (iii) la insuficiencia de cualquier otro órgano o sistema distintos de los anteriores así constatado con los datos de la historia clínica.

- Desnutrición. Variable medida usando los valores de la albúmina en la analítica de sangre realizada en urgencias 0 , en su defecto, en la primera analítica realizada durante el ingreso; se ha valorado como (0) en los casos de albúmina normal (mayor de 3,5 g/dl) y como (1) en los casos en los que la albúmina fuera igual o inferior a 3,5 g/dl, sin diferenciar si ésta desnutrición era leve, moderada o severa.

-Intervenciones agresivas

Se ha considerado intervención agresiva cualquier tipo de intervencionismo, ya sea diagnóstico o terapéutico, entre los que incluimos: Endoscopias, alimentación parenteral, canalizaciones vasculares, biopsias, etc. diferentes al cateterismo urinario que ya se ha estudiado previamente. Se trata de procedimientos frecuentes y muchas veces evitables en ancianos hospitalizados. Se van a clasificar según se hayan realizado (1) o no (0) alguna de las citadas intervenciones sobre nuestro paciente a lo largo del ingreso.

\section{-Medicación}

Los efectos secundarios de los medicamentos, en especial de los de efecto anticolinérgico, son la principal causa del cuadro confusional agudo en el anciano21 pero a pesar de esto, no hay muchos estudios que evalúen cada fármaco en concreto y cada estudio utiliza distintos sistemas para evaluar la asociación que tiene la medicación en la aparición de delirium. A la conclusión a la que han llegado la mayoría de trabajos es que la polifarmacia se considera un factor de riesgo independiente22, como también concluyen S. Ahmed y Colaboradores19, así como la existencia de familias farmacológicas especialmente peligrosas así como que el uso de neurolépticos o benzodiacepinas en el momento del ingreso se asocia con delirium en análisis univariables.

A la vista de estos resultados, se distinguen dentro de la categoría "medicación" siete variables distintas:

1. Polifarmacia, definida como la toma de tres o más medicamentos, según esté especificado como tratamiento actual en la anamnesis realizada en el momento del ingreso.

2. El uso crónico de uno o más de los fármacos considerados de alto riesgo, entre los que se encuentran los psicotropos (benzodiacepinas, antipsicóticos o neurolépticos, etc.), anticolinérgicos, antiarrítmicos cardíacos etc.

3. Si entre los fármacos de riesgo que toma de forma crónica se encuentran las benzodiacepinas, pues merece la pena estudiar concretamente el riesgo de su uso, al ser de los medicamentos más fuertemente asociados.

4. Si entre los fármacos de riesgo que toma de forma crónica se encuentran los neurolépticos.

5. Si entre los fármacos de riesgo que toma de forma crónica se encuentra algún fármaco con efecto anticolinérgico central demostrado. 
6. Si el fármacos de riesgo que toma de forma crónica es distinto de los anteriores.

7. Si se han añadido fármacos de riesgo durante la hospitalización.

\section{Métodos estadísticos}

Para el estudio de las variables discretas se ha utilizado el test de la Chi-Cuadrado y el estadístico exacto de Fisher; además se han calculado los Odds Ratio (OR) y los intervalos de confianza al 95\% (IC) para las variables consideradas factores de riesgo para síndrome confusional agudo. Se han analizado las variables continuas índice Apache II e índice de Charlsson mediante el test T de Student tras comprobar su distribución normal. No se identificaron posibles factores de confusión por lo que no se empleó ninguna herramienta para su control.

\section{RESULTADOS}

En la Tabla 1 se observa que la variable sexo masculino muestra un riesgo de síndrome confusional agudo a nivel muestral con una odds ratio $>1$, aunque dicho resultado no es significativo $(p>0,05)$ por tanto no se puede afirmar que sea un factor de riesgo significativamente asociado a síndrome confusional agudo en el contexto de este análisis. Para medir si a mayor edad existe mayor riesgo de SCA, se ha elegido como punto de corte los 75 años, como se ha observado en los estudios de la literatura que se han revisado. Análogamente que con la variable de género, sucede con la variable edad mayor de 75 años, que a pesar de tener una odds ratio de 2,5 , no se puede sostener que la misma sea un factor de riesgo significativamente asociado a síndrome confusional agudo en el contexto de este análisis ( $p>0,05)$.

\begin{tabular}{|c|c|c|c|c|c|c|}
\hline \multirow{2}{*}{\multicolumn{2}{|c|}{$\begin{array}{c}\text { FACTORES } \\
\text { DEMOGRAFICOS }\end{array}$}} & \multicolumn{2}{|c|}{ Delirium } & \multirow{4}{*}{$\frac{\text { OR. }}{1,33}$} & & \\
\hline & & No & Sí & & \multicolumn{2}{|c|}{ Intervalo de Confianze } \\
\hline \multirow{2}{*}{ Sexo } & Mujer & 12 & 10 & & \multirow{2}{*}{0,47} & \multirow{2}{*}{3,82} \\
\hline & Hombre & 18 & 20 & & & \\
\hline \multirow{2}{*}{ Edad } & $<75$ años & 10 & 5 & \multirow{2}{*}{2,5} & \multirow{2}{*}{0,74} & \multirow{2}{*}{8,50} \\
\hline & $>75$ años & 20 & 25 & & & \\
\hline
\end{tabular}

Significación de la chi cuadrado: ${ }_{*}^{*}: p_{<}<0,05 ; * *: p<0,01$

Tabla 1. Factores demográficos

En el análisis se observa (Tabla 2) que la variable depresión muestra un riesgo de síndrome confusional agudo a nivel muestral con una odds ratio $>1$, aunque dicho resultado no es significativo $(p>0,05)$ por tanto no se puede afirmar que sea un factor de riesgo significativamente asociado a síndrome confusional agudo en el contexto de este análisis.

Igualmente se observa con el deterioro cognitivo, no se puede afirmar que esta variable sea un factor de riesgo significativamente asociado a síndrome confusional agudo en el contexto de este análisis (Tabla 2).

Por lo que respecta a otras alteraciones mentales muestra un riesgo de síndrome confusional agudo a nivel muestral con una odds ratio $>1$, aunque dicho resultado no es significativo $(p>$ 
0,05) por tanto no se puede afirmar que sea un factor de riesgo significativamente asociado a síndrome confusional agudo en el contexto de este análisis.

En cuanto a la variable estado mental alterado, tiene un riesgo de síndrome confusional agudo a nivel muestral pues presenta una odds ratio de 7,25 que significa que el paciente que presenta dos o más de las alteraciones mentales estudiadas tiene 7,25 veces más riesgo de desarrollar un delirium que el paciente que no las presenta; resultado que es estadísticamente significativo ( $p<$ $0,05)$ lo que confirma que esta variable es un factor de riesgo significativamente asociado a síndrome confusional agudo en el contexto de este análisis, resultado que debe ser tratado con cautela puesto que el intervalo de confianza al $95 \%<1$ (Tabla 2).

\begin{tabular}{|c|c|c|c|c|c|c|}
\hline \multirow{2}{*}{\multicolumn{2}{|c|}{ ESTADO MENTAL }} & \multicolumn{2}{|c|}{ Delirium } & \multirow[b]{2}{*}{ OR. } & & \\
\hline & & \multirow{2}{*}{$\begin{array}{l}\text { No } \\
25\end{array}$} & \multirow{2}{*}{$\begin{array}{l}\text { Sí } \\
23\end{array}$} & & \multicolumn{2}{|c|}{ Intervalo de Confianza } \\
\hline \multirow{2}{*}{ Depresión } & No & & & \multirow{2}{*}{1,52} & \multirow{2}{*}{0,42} & \multirow{2}{*}{5,47} \\
\hline & Sí & 5 & 7 & & & \\
\hline \multirow{2}{*}{$\begin{array}{l}\text { Deterioro } \\
\text { Cognitivo }\end{array}$} & $\mathrm{No} /$ leve & 22 & 19 & \multirow{2}{*}{1,60} & \multirow{2}{*}{0,53} & \multirow{2}{*}{4,78} \\
\hline & Sí/severo & 8 & 11 & & & \\
\hline \multirow{2}{*}{$\begin{array}{c}\text { Otras } \\
\text { alteraciones } \\
\text { mentales }\end{array}$} & No & 28 & 25 & \multirow{2}{*}{2,8} & \multirow{2}{*}{0,50} & \multirow{2}{*}{15,73} \\
\hline & Sí & 2 & 5 & & & \\
\hline \multirow{2}{*}{$\begin{array}{l}\text { Estado } \\
\text { mental }\end{array}$} & $\begin{array}{c}\text { No } \\
\text { alterado }\end{array}$ & 29 & 24 & \multirow{2}{*}{$7,25^{*}$} & \multirow{2}{*}{0,82} & \multirow{2}{*}{64,46} \\
\hline & Alterado & 1 & 6 & & & \\
\hline
\end{tabular}

Significación de la chi cuadrado * ${ }^{*}$ p $<0,05 ;{ }^{* *}: p<0,01$

Tabla 2. Estado mental

En el análisis se observa (Tabla 3) que a mayor severidad de la enfermedad, medida mediante la escala Apache II, existe mayor riesgo de síndrome confusional agudo y además este riesgo es estadísticamente significativo, como indica el valor de $\mathrm{p}<0,01$; por tanto se puede afirmar que esta variable es un factor de riesgo asociado a síndrome confusional agudo en el contexto de este análisis.

En cuanto a la variable referente a la comorbilidad, medida mediante el índice de Charlson, se observa que a nivel muestral existe diferencia entre casos y controles, pues los casos presentan un mayor índice de comorbilidad, pero esta diferencia no es significativa ( $p>0,05)$; y por tanto se puede considerar que esta variable sea un factor de riesgo asociado a síndrome confusional agudo en el contexto de este análisis (Tabla3). 


\begin{tabular}{|c|c|c|c|c|c|c|}
\hline ENF. FÍSICA & Delirium & Media & $\begin{array}{c}\text { Desviación } \\
\text { típica }\end{array}$ & Mediana & $\begin{array}{c}\text { Percentil } \\
25-75\end{array}$ & P-valor \\
\hline \multirow{2}{*}{ Apache II } & Controles & 9,5 & 4,3 & 8 & $6,8-11,5$ & \multirow{2}{*}{0,001} \\
\cline { 2 - 6 } & Casos & 13,9 & 5,3 & 13,5 & $10-17$ & \\
\hline \multirow{3}{*}{ I. Charlson } & Controles & 6,4 & 2,7 & 6,2 & $4,6-7,7$ & \multirow{2}{*}{0,22} \\
\cline { 2 - 6 } & Casos & 7,3 & 3,2 & 6,9 & $5,1-8,8$ & \\
\hline
\end{tabular}

*Prueba de T de Student

Tabla 3. Severidad de la enfermedad (Apache II) e índice de comorbilidad (I. Charlson)

En la Tabla 4 se observa que la situación funcional previa muestra un riesgo de síndrome confusional agudo a nivel muestral con una odds ratio $>1$, aunque dicho resultado no es significativo $(p>0,05)$ por tanto no se puede afirmar que sea un factor de riesgo significativamente asociado a síndrome confusional agudo en el contexto de este análisis.

Por lo que respecta a la variable historia previa de delirium (Tabla 4) muestra riesgo de síndrome confusional agudo a nivel muestral lo que se traduce en un odds ratio $>1$; con una $p<0,05$ que nos permite afirmar que esta variable es factor de riesgo significativamente asociado a síndrome confusional agudo en el contexto de este análisis.

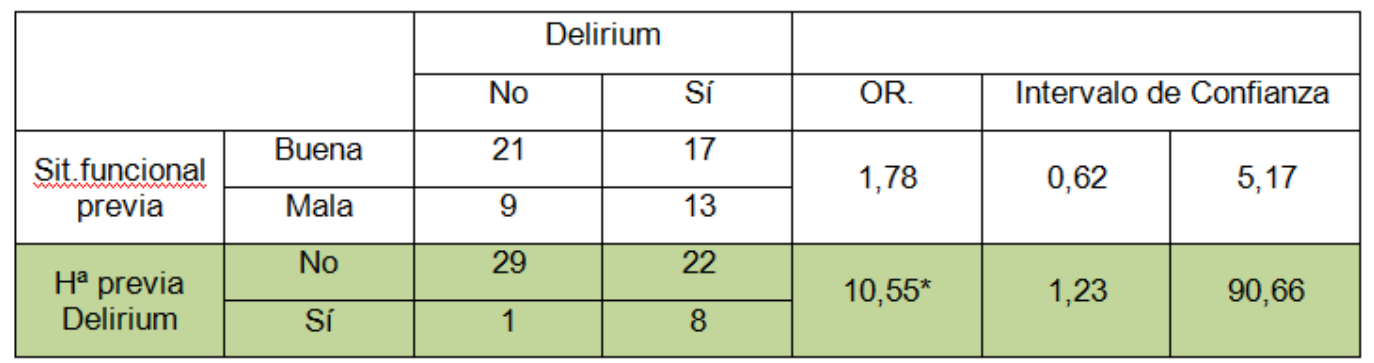

Significación de la chi cuadrado ${ }_{*}^{*}: p<0,05 ;{ }^{* *}: p<0,01$

Tabla 4. Situación funcional previa e historia previa de delirium

En la Tabla 5 se observa que la deprivación sensorial muestra un riesgo de síndrome confusional agudo a nivel muestral con una odds ratio $>1$, aunque dicho resultado no es significativo $(p>$ $0,05)$ por tanto no se puede afirmar que sea un factor de riesgo significativamente asociado a síndrome confusional agudo en el contexto de este análisis.

En cuanto a la variable catéter urinario, muestra riesgo de síndrome confusional agudo a nivel muestral lo que se traduce en un odds ratio $>1$; con una $p<0,05$ que nos permite afirmar que la misma es un factor de riesgo significativamente asociado a síndrome confusional agudo en el contexto de este análisis (Tabla 5) . 
Así mismo se observa en la Tabla 5 que la inmovilidad expresa riesgo de síndrome confusional agudo a nivel muestral pues presenta una odds ratio $>1$; pero tiene una $p>0,05$ y por tanto no se puede afirmar que esta variable sea un factor de riesgo significativamente asociado a síndrome confusional agudo en el contexto de este análisis.

Por lo que respecta a las limitaciones del estado físico, esta variable muestra riesgo de síndrome confusional agudo a nivel muestral lo que se traduce en un odds ratio $>1$, y presenta además una $p<0,05$; lo que confirma que esta variable es factor de riesgo significativamente asociado a síndrome confusional agudo en el contexto de este análisis.

\begin{tabular}{|c|c|c|c|c|c|c|}
\hline \multirow{2}{*}{\multicolumn{2}{|c|}{ ESTADO FÍSICO }} & \multicolumn{2}{|c|}{ Delirium } & \multirow{4}{*}{$\begin{array}{c}\text { OR. } \\
0,8\end{array}$} & & \\
\hline & & \multirow{2}{*}{$\begin{array}{l}\text { No } \\
24\end{array}$} & \multirow{2}{*}{$\begin{array}{l}\text { Sí } \\
25\end{array}$} & & \multicolumn{2}{|c|}{ Intervalo de Confianza } \\
\hline \multirow{2}{*}{$\begin{array}{c}\text { Deprivación } \\
\text { sensorial }\end{array}$} & No & & & & & 70 \\
\hline & Sí & 6 & 5 & & 0,22 & $2, \nabla r$ \\
\hline \multirow{2}{*}{$\begin{array}{l}\text { Catéter } \\
\text { urinario }\end{array}$} & No & 26 & 19 & \multirow{2}{*}{$3,76^{*}$} & \multirow{2}{*}{1,04} & \multirow{2}{*}{13,65} \\
\hline & Sí & 4 & 11 & & & \\
\hline \multirow{2}{*}{ Inmovilidad } & No & 20 & 13 & \multirow{2}{*}{2,62} & \multirow{2}{*}{0,92} & \multirow{2}{*}{7,46} \\
\hline & Sí & 10 & 17 & & & \\
\hline \multirow{2}{*}{$\begin{array}{c}\text { Limitaciones } \\
\text { E. físico }\end{array}$} & No/leves & 27 & 19 & \multirow{2}{*}{$5,21^{*}$} & \multirow{2}{*}{1,28} & \multirow{2}{*}{21,24} \\
\hline & Sí/severas & 3 & 11 & & & \\
\hline
\end{tabular}

Significación de la chi cuadrado ${ }_{*}^{*}: \mathrm{p}<0,05 ;{ }^{* *}: \mathrm{p}<0,01$

\section{Tabla 5. Estado físico}

En la Tabla 6 se observa que la variable infección muestra un riesgo de síndrome confusional agudo a nivel muestral con una odds ratio $>1$, resultado que es significativo $(p<0,05)$ por tanto se puede afirmar que es un factor de riesgo significativamente asociado a síndrome confusional agudo en el contexto de este análisis.

Análogamente sucede con la variable referente a la alteración de la natremia, que muestra un riesgo de síndrome confusional agudo a nivel muestral con una odds ratio $>1$, resultado que es significativo $(p<0,05)$ por tanto se puede afirmar que es un factor de riesgo significativamente asociado a síndrome confusional agudo en el contexto de este análisis (Tabla 6).

Igualmente se observa con la anemia, que muestra un riesgo a nivel muestral con una odds ratio > 1 , resultado que es significativo $(p<0,05)$ por tanto se puede afirmar que es un factor de riesgo significativamente asociado a síndrome confusional agudo en el contexto de este análisis (Tabla 6). 
Por lo que respecta a la variable fiebre, se observa en la Tabla 6 que muestra un riesgo de SCA a nivel muestral con una odds ratio $>1$, aunque dicho resultado no es significativo $(p>0,05)$ por tanto no se puede afirmar que sea un factor de riesgo significativamente asociado a síndrome confusional agudo en el contexto de este análisis.

En cuanto a las variables referidas al dolor y a la presencia de enfermedad neurológica primaria, ninguna de las dos muestra riesgo de SCA a nivel muestral (odds ratio < 1 ) y por tanto no se puede corroborar que se traten de factores de riesgo asociados a síndrome confusional agudo en el contexto de este análisis.

\begin{tabular}{|c|c|c|c|c|c|c|}
\hline \multirow{2}{*}{\multicolumn{2}{|c|}{$\begin{array}{c}\text { PATOLOGÍA } \\
\text { INTERCURRENTE } 1\end{array}$}} & \multicolumn{2}{|c|}{ Delirium } & \multirow[b]{2}{*}{ OR. } & & \\
\hline & & No & Sí & & \multicolumn{2}{|c|}{ Intervalo de Confianza } \\
\hline \multirow{2}{*}{ Infección } & No & 16 & 8 & \multirow{2}{*}{$3,14^{*}$} & \multirow{2}{*}{1,07} & \multirow{2}{*}{9,27} \\
\hline & Sí & 14 & 22 & & & \\
\hline \multirow{2}{*}{$\begin{array}{c}\text { Alteración } \\
\mathrm{Na}^{+}\end{array}$} & No & 27 & 20 & \multirow{2}{*}{$4,5^{\star}$} & \multirow{2}{*}{1,10} & \multirow{2}{*}{18,50} \\
\hline & Sí & 3 & 10 & & & \\
\hline \multirow{2}{*}{ Anemia } & No & 22 & 11 & \multirow{2}{*}{$4,75^{* *}$} & \multirow{2}{*}{1,58} & \multirow{2}{*}{14,25} \\
\hline & Sí & 8 & 19 & & & \\
\hline \multirow{2}{*}{ Fiebre } & No & 22 & 19 & \multirow{2}{*}{1,59} & \multirow{2}{*}{0,53} & \multirow{2}{*}{4,78} \\
\hline & Sí & 8 & 11 & & & \\
\hline \multirow{2}{*}{ Dolor } & No & 22 & 24 & \multirow{2}{*}{0,69} & \multirow{2}{*}{0,21} & \multirow{2}{*}{2,30} \\
\hline & Sí & 8 & 6 & & & \\
\hline \multirow{2}{*}{$\begin{array}{c}\text { Enf. } \\
\text { neurológ. } 1^{\mathrm{a}}\end{array}$} & No & 28 & 29 & \multirow{2}{*}{0,48} & \multirow{2}{*}{0,04} & \multirow{2}{*}{5,63} \\
\hline & Sí & 2 & 1 & & & \\
\hline
\end{tabular}

Significación de la chi cuadrado *: $p<0,05 ;$ **: $p<0,01$

Tabla 6. Patología intercurrente 1

En la Tabla 7 se observa que la variable desnutrición muestra un riesgo de SCA a nivel muestral con una odds ratio $>1$, resultado que es significativo $(p<0,05)$ por tanto se puede afirmar que es un factor de riesgo significativamente asociado a síndrome confusional agudo en el contexto de este análisis.

Análogamente ocurre con la variable insuficiencia renal, que muestra un riesgo de SCA a nivel muestral con una odds ratio $>1$, resultado que es significativo $(p<0,01)$ por tanto se puede afirmar que es un factor de riesgo significativamente asociado a síndrome confusional agudo en el contexto de este análisis (Tabla 7).

Por lo que respecta a las variables insuficiencia respiratoria aguda y de otros órganos distintos de los anteriores, se observa en la Tabla 7 que aunque muestran un riesgo de SCA a nivel muestral con una odds ratio $>1$, no es significativo $(p>0,05)$ por tanto no se puede afirmar que sean factores de riesgo significativamente asociados a síndrome confusional agudo en el contexto de este análisis. 
En cuanto a la variable intervención agresiva, tiene un riesgo de SCA a nivel muestral con una odds ratio > 1 ; resultado que, sin embargo, no es significativo $(p>0,05)$ lo que no nos permite afirmar que esta variable sea un factor de riesgo significativamente asociado a síndrome confusional agudo en el contexto de este análisis (Tabla 7).

\begin{tabular}{|c|c|c|c|c|c|c|}
\hline \multirow{2}{*}{\multicolumn{2}{|c|}{$\begin{array}{c}\text { PATOLOGÍA } \\
\text { INTERCURRENTE } 2\end{array}$}} & \multicolumn{2}{|c|}{ Delirium } & \multirow{4}{*}{$\begin{array}{c}\text { OR. } \\
3,67^{*}\end{array}$} & & \\
\hline & & No & Sí & & \multicolumn{2}{|c|}{ Intervalo de Confianza } \\
\hline \multirow{2}{*}{ Desnutrición } & No & 12 & 6 & & \multirow{2}{*}{1,10} & \multirow{2}{*}{12,25} \\
\hline & Sí & 12 & 22 & & & \\
\hline \multirow{2}{*}{$\begin{array}{l}\text { Insuficiencia } \\
\text { respiratoria }\end{array}$} & No & 19 & 15 & \multirow{2}{*}{1,73} & \multirow{2}{*}{0,62} & \multirow{2}{*}{4,85} \\
\hline & Sí & 11 & 15 & & & \\
\hline \multirow{2}{*}{$\begin{array}{l}\text { Insuficiencia } \\
\text { renal }\end{array}$} & No & 26 & 15 & \multirow{2}{*}{$6,50^{\star \star}$} & \multirow{2}{*}{1,82} & \multirow{2}{*}{23,21} \\
\hline & Sí & 4 & 15 & & & \\
\hline \multirow{2}{*}{$\begin{array}{l}\text { Insuficiencia } \\
\text { otros órg. }\end{array}$} & No & 23 & 22 & \multirow{2}{*}{1,20} & \multirow{2}{*}{0,37} & \multirow{2}{*}{3,85} \\
\hline & Sí & 7 & 8 & & & \\
\hline \multirow{2}{*}{$\begin{array}{l}\text { Intervención } \\
\text { agresiva }\end{array}$} & No & 19 & 12 & \multirow{2}{*}{2,60} & \multirow{2}{*}{0,91} & \multirow{2}{*}{7,34} \\
\hline & Sí & 11 & 18 & & & \\
\hline
\end{tabular}

Significación de la chi cuadrado $*$ *: $p<0,05 ;{ }^{* *}: p<0,01$

Tabla 7. Patología intercurrente 2

En la Tabla 8 se observa que la polifarmacia, a pesar de tener una odds ratio $>1$ a nivel muestral, no es un factor de riesgo significativamente asociado a síndrome confusional agudo en el contexto de este análisis $(p>0,05)$.

En cuanto al uso crónico de medicamentos de alto riesgo, se observa en la Tabla 8 que, estudiados conjuntamente, muestran un riesgo de delirium con una odds ratio de 6,88 $(p<0,01)$; resultado estadísticamente significativo que permite afirmar que esta variable está asociada a síndrome confusional agudo en el contexto de este análisis. Por el contrario, analizando por separado los distintos fármacos se observa que sólo los neurolépticos se asocian de forma significativa $(p<0,05)$ al síndrome confusional agudo, mientras que las benzodiacepinas, los anticolinérgicos y otros medicamentos de riesgo no tienen resultados significativos $(p>0,05)$.

Para terminar, por lo que respecta a la medicación añadida durante el ingreso de riesgo, tiene un riesgo de delirium a nivel muestral con una odds ratio $>1$, resultado que es significativo $(p<0,01)$, por tanto se puede afirmar que es un factor de riesgo significativamente asociado a síndrome confusional agudo en el contexto de este análisis (Tabla 8). 


\begin{tabular}{|c|c|c|c|c|c|c|}
\hline \multirow{2}{*}{\multicolumn{2}{|c|}{ MEDICACIÓN }} & \multicolumn{2}{|c|}{ Delirium } & \multirow{4}{*}{$\begin{array}{c}\text { OR. } \\
1,8\end{array}$} & & \\
\hline & & \multirow{2}{*}{$\begin{array}{l}\text { No } \\
5\end{array}$} & \multirow{2}{*}{$\begin{array}{l}\text { Sí } \\
3\end{array}$} & & \multicolumn{2}{|c|}{ Intervalo de Confianza } \\
\hline \multirow{2}{*}{ Polifarmacia } & No & & & & & \\
\hline & Sí & 25 & 27 & & 0,00 & $0,0<$ \\
\hline \multirow{2}{*}{$\begin{array}{l}\text { Medicación alto } \\
\text { riesgo }\end{array}$} & No & 13 & 3 & \multirow{2}{*}{$6,88^{* *}$} & \multirow{2}{*}{1,70} & \multirow{2}{*}{27,75} \\
\hline & Sí & 17 & 27 & & & \\
\hline \multirow{2}{*}{$\begin{array}{c}\text { Uso de } \\
\text { Benzodiacepinas }\end{array}$} & No & 20 & 13 & \multirow{2}{*}{2,62} & \multirow{2}{*}{0,92} & \multirow{2}{*}{7,46} \\
\hline & Sí & 10 & 17 & & & \\
\hline \multirow{2}{*}{$\begin{array}{c}\text { Uso de } \\
\text { neurolépticos }\end{array}$} & No & 28 & 22 & \multirow{2}{*}{$5,10^{*}$} & \multirow{2}{*}{0,98} & \multirow{2}{*}{26,43} \\
\hline & Sí & 2 & 8 & & & \\
\hline \multirow{2}{*}{$\begin{array}{c}\text { Uso de } \\
\text { anticolinérgicos }\end{array}$} & No & 28 & 23 & \multirow{2}{*}{4,26} & \multirow{2}{*}{0,81} & \multirow{2}{*}{22,53} \\
\hline & Sí & 2 & 7 & & & \\
\hline \multirow{2}{*}{$\begin{array}{l}\text { Uso de otros ttos } \\
\text { de riesgo }\end{array}$} & No & 19 & 15 & \multirow{2}{*}{1,73} & \multirow{2}{*}{0,62} & \multirow{2}{*}{4,85} \\
\hline & Sí & 11 & 15 & & & \\
\hline \multirow{2}{*}{$\begin{array}{l}\text { Medicación } \\
\text { añadida de } \\
\text { riesgo }\end{array}$} & No & 27 & 18 & \multirow{2}{*}{$6^{* \star}$} & \multirow{2}{*}{1,48} & \multirow{2}{*}{24,30} \\
\hline & Sí & 3 & 12 & & & \\
\hline \multicolumn{7}{|c|}{ Significación de la chi cuadrado ${ }^{*}: \mathrm{p}<0,05 ; * *: p<0,01$} \\
\hline \multicolumn{7}{|c|}{ Tabla 8. Medicación } \\
\hline
\end{tabular}

\section{DISCUSIÓN}

De entre los factores de riesgo que se han estudiado en este trabajo, se han identificado trece de ellos como estadísticamente significativos: severidad de la enfermedad; historia previa de Delirium (OR 10,6); alteración del estado mental (OR 7,3); medicación crónica de alto riesgo (OR 6,9); insuficiencia renal (OR 6,5); medicación añadida de riesgo (OR 6); limitaciones del estado físico (OR 5,2); uso crónico de neurolépticos (OR 5,1); anemia (OR 4,75); alteraciones de la natremia (OR 4,5); catéter urinario (OR 3,8); desnutrición (OR 3,7); infección (OR 3,1). Estos resultados confirman lo que estudios previos, entre ellos el metaanálisis de S. Ahmed y colaboradores19 , ya habían evidenciado con otras muestras y nos permiten afirmar que hay asociación entre ellos y la aparición de síndrome confusional agudo en nuestra población. Es necesario puntualizar que los intervalos de confianza (que permiten estimar entre qué valores está el valor real de la población a partir del que podemos obtener de nuestra muestra) para estos valores de OR son amplios, puesto que el tamaño de nuestra muestra limita su potencia estadística.

Así mismo, se han encontrado otros tres factores de riesgo que están cerca de ser considerados significativos y que, presumimos, podrían obtener resultados de mayor fuerza estadística en estudios con mayor tamaño muestral; son los siguientes: uso crónico de benzodiacepinas (OR 2,6); intervención agresiva (OR 2,6); inmovilidad (OR 2,6).

Otros factores de riesgo de reconocida importancia en la literatura, sin embargo, no han podido ser corroborados en este análisis, entre los más importantes se encuentran: la edad avanzada, el deterioro cognitivo estudiado de forma aislada, el índice de comorbilidad, la situación funcional previa y la polifarmacia.

En lo referente a la edad, ya habíamos comentado que en la revisión sistemática realizada por $\mathrm{S}$. Ahmed y Colaboradores, fue el factor de riesgo más estudiado, pues nueve de los once artículos incluidos lo analizaban, y de éstos, cuatro demostraron una asociación estadísticamente 
significativa. En nuestro análisis, a pesar de que la edad media de los casos es de 80,2 y de los controles de 77,7, no existen grandes diferencias entre la edad que presentan unos y otros, hecho que puede explicarse por el aumento de la edad media de los pacientes ingresados en medicina interna, cada vez más ancianos, y a que en este estudio se trabaja con una muestra limitada a 60 pacientes.

El deterioro cognitivo o la demencia es otro de los factores que a pesar de ser ampliamente reconocido como factor de riesgo y ser evaluado y utilizado para prever la aparición de SCA, no tiene resultados significativos estudiado de forma aislada en este trabajo. Esto puede estar justificado por muchos motivos; en seis de los siete estudios incluidos en el meta-análisis de $\mathrm{S}$. Ahmed y Colaboradores19, el deterioro cognitivo se evalúa mediante el MMSE (mini mental state examination), una misma escala con la que se consigue medir de forma objetiva el grado de deterioro presente en los pacientes, sin embargo, debido a las características de nuestro trabajo esta evaluación ha sido llevada a cabo de forma subjetiva por medio de las notas incluidas en la anamnesis referentes al estado mental del paciente y, por tanto, no existe un manera clara, sistematizada y unificada de valorar su grado. Es por esto que pese a no haber conseguido demostrar su asociación, creemos justificado que pueda valorarse como un factor de riesgo de importancia por su capacidad de predicción de delirium, siempre que se usen test clínicos como el MMSE o el CDT (Clock drawing test)23.

Con respecto a la comorbilidad que presenta el paciente, medida mediante el Índice de Charlson, existe apenas diferencia entre el valor medio que presentan los casos y los controles. A pesar de mostrar una asociación significativa en estudios como el de Villalpando- Berumen y Colaboradores 24 , esta asociación no muestra una gran fuerza estadística (OR 1,16) lo que nos hace pensar que no existen grandes diferencias entre las co-morbilidades que presentan unos y otros, si tenemos en cuenta que son pacientes de edad avanzada que de por sí, van a presentar gran número de patologías.

Sí que muestra una asociación estadísticamente significativa en la literatura la variable referente a la situación funcional previa del paciente, con una OR de 8,4 en el estudio de Wakefield BJ.25 en 2002 incluido en la revisión sistemática de S. Ahmed et al.18 Que este resultado no pueda ser corroborado en nuestro análisis puede deberse, entre otras razones, al hecho de que la medición de este parámetro en nuestro estudio haya sido realizada de manera subjetiva a través de las anotaciones del equipo médico acerca del grado de dependencia del paciente, y no por medio de una escala numérica y objetiva como es el índice de Barthel (Autonomía para las actividades de la vida diaria) así aceptado por la comunidad científica para objetivarlo.

En cuanto a la polifarmacia, que en nuestro análisis no ha mostrado una asociación estadísticamente significativa con el síndrome confusional agudo, sí lo ha hecho en cuatro de los seis estudios incluidos en la revisión de S. Ahmed et al. 18 donde se estudiaba. Los resultados obtenidos en nuestro trabajo no muestran una gran diferencia entre la polifarmacia en casos y en controles, dato que puede explicarse por el hecho de que actualmente la mayoría de personas ancianas toman más de 3 fármacos diarios de forma crónica, lo que no permite discriminar entre unos y otros en lo referente a este factor de riesgo. 


\section{CONCLUSIONES}

Las fortalezas de este estudio son que un gran número de factores de riesgo hayan podido ser estudiados, así como el haber incluido no sólo los delirium incidentes si no también aquellos que ya estaban presentes en el momento del ingreso. Se han excluido los síndromes confusionales acontecidos tras una intervención quirúrgica, pues se ha considerado que, aunque presentan factores comunes otros factores de riesgo que presentan son específicos y en muchas ocasiones, distintos de los presentes en pacientes con patologías médicas.

Entre las limitaciones que presenta este trabajo, se encuentran las intrínsecamente relacionadas con el tipo de estudio (de casos y controles) realizado, como son: que no pueda establecerse la secuencia de eventos de interés, que no puedan proporcionar estimadores de prevalencia, incidencia o riesgo atribuible o los posibles errores en la selección de casos y controles. En lo relativo a este último punto, existen además limitaciones debidas al diseño de este estudio, dado que la identificación de los casos se ha realizado a partir de las interconsultas a psiquiatría que terminaron por ser diagnosticadas de Delirium; esto implica un posible sesgo de inclusión, pues no todos los pacientes que han sufrido un síndrome confusional agudo han necesitado de una interconsulta al especialista para su control, tan sólo los casos de mayor dificultad en su manejo, siendo los únicos susceptibles de ser incluidos en el grupo de casos. Así mismo, el hecho de que el diagnóstico sea clínico y no de laboratorio puede haber supuesto que algunos individuos hayan sido clasificados como casos cuando no lo eran. Se trataría de un sesgo de diagnóstico.

En conclusión este trabajo ha intentado servir de estudio exploratorio para identificar y confirmar los factores de riesgo de desarrollo del síndrome confusional agudo en nuestro medio, el Hospital Universitario Reina Sofía de Murcia. Incluso con las limitaciones metodológicas mencionadas más arriba, ciertos factores de riesgo parecen consistentes, algunos de ellos son fijos (severidad de la enfermedad, historia previa de SCA, alteración del estado mental, limitaciones del estado físico) pero otros son potencialmente modificables (medicación crónica de alto riesgo, insuficiencia renal, medicación añadida de riesgo, uso crónico de neurolépticos, anemia, alteraciones de la natremia, catéter urinario, desnutrición, infección) y podrían permitir una intervención clínica para prevenir la aparición de este trastorno. Ciertos factores de riesgo como son el índice de comorbilidad y la polifarmacia, requerirán estudios más amplios para una mejor definición de su papel en la aparición del síndrome confusional agudo. 


\section{REFERENCIAS}

Ahmed S, Leurent B, Sampson E.Risk factors for incident delirium among older people in acute hospital medical units: a systematic review and meta-analysis. Age Ageing. 2014; 43:326-333. DOI 10.1093/ageing/afu022

American Psychriatic Association.Diagnostic and Statistic Manual of Mental Disorders DSM-5. Washington DC: APA; 2013.

American Psychriatic Association. Ganuza A, González-Torres MA, Gaviria M. El Delirium: Una revisión orientada a la práctica clínica. Rev. Asoc. Esp. Neuropsiq. 2012; 32(114):247-259. DOI 10.4321/S0211-57352012000200003

Carrasco M, Villarroel L, Calderón J, Martínez G, Andrade M, González T.Riesgo de delirium durante la hospitalización en personas mayores: desarrollo y validación de un modelo de predicción clínica. Rev. méd. Chile. 2014; 142(7):826-832. https://scielo.conicyt.cl/scielo.php?script=sci_ar

Curyto KJ, Johnson J, TenHave T, Mossey J, Knott K, Katz IR.Survival of hospitalized elderly patients with delirium. Am J Geriatr Psychiatry. 2001; 9:141-147. DOI 10.1016/j.revmed.2007.04.004

Formiga F, San José A, López-Soto A, Ruiz D, Urrutia A, Duaso E.Prevalencia del delirium en pacientes ingresados por enfermedad médica. Med Clin (Barc). 2007;129:571-573. DOI 10.1093/ageing/afu022

Gentric A, Le Deun P, Estivin S.Prévention du syndrome confusionnel dans un service de médecine interne gériatrique. Rev Med Interne. 2007; 28(9):589- 593. DOI 10.1016/j.revmed.2007.04.004

González M, Martínez G, Calderon J, Villarroel L, Yuri F, Rojas C, et al.Impact of delirium on shortterm mortality in elderly inpatients: a prospective cohort study. Psychosomatics. 2009; 50(3):234238. DOI 10.1176/appi.psy.50.3.234

Inouye SK, Charpentier PA.Precipitating factors for delirium in hospitalized elderly persons. Predictive model and interrelationship with baseline vulnerability. JAMA. 1996; 275(11): 852-857. PubMed

Inouye SK, Foreman MD, Mion LC, Katz KH, Cooney LM.Nurse's recognition of delirim and its symptoms: comparison of nurse and researcher ratings. Arch Intern Med. 2001; 161:2467-2473. PubMed

Inouye SK, Rushing JT, Foreman MD, Palmer RM, Pom- pei P.Does delirium contribute to poor hospital outcomes? A three-site epidemiologic study. J Gen Intern Med. 1998; 13(4):234-242. PubMed

Inouye SK, Viscoli CM, Horwitz RI, Hurst LD, Tinetti ME.A predictive model for delirium in hospitalized elderly medical patients based on admission characteristics. Ann Intern Med. 1993; 119(6): 474481. PubMed

Kaufman DW, Kelly JP, Rosemberg L, Anderson TE, Mitchell AA.Recent patterns of medication use in the ambulatory adult population in the United States. JAMA. 2002; 287:337-344. 
PubMed

Lázaro M, Ribera J.Síndrome confusional (delirium) en el anciano. Psicogeriatría. 2009; 1(4):209-221.

DOI 10.1093/ageing/afu022

Leslie DL, Zhang Y, Bogardus ST, Holford TR, Leo-Summers LS, Inouye SK.Consequences of preventing delirium in hospitalized older adults on nursing home costs. J Am Geriatr Soc. 2005; 53: 405-409. PubMed

McCusker J, Cole M, Dendukuri N, Belzile E, Primeau F.Delirium in older medical inpatients and subsequent cognitive and functional status: a prospective study. CMAJ. 2001; 65(5):575-583.

PubMed

Medical Chin YC, Koh GCH, Tay YK, Tan CH, Merchant RA.Underdiagnosis of delirium on admission and prediction of patients who will develop delirium during their inpatient stay: a pilot study. Singapore Med J. 2016; 57(1):18-21 DOI 10.11622/smedj.2016007

Morandi P, Pandharipande M, Trabucchi R, Rozzini G, Mistraletti AC, Trompeo.Understanding international differences in terminology for delirium and other types of acute brain dysfunction in critically ill patients. Intensive Care Med. 2008; 34:1907-1915 DOI 10.1007/s00134-008-1177-6

O'Keeffe ST, Lavan JN.Predicting delirium in elderly patients: development and validation of a riskstratification model. Age Ageing. 1996; 25(4): 317-321. PubMed

Pompei P, Foreman M, Rudberg MA, Inouye SK, Braund V, Cassel CK.Delirium in hospitalized older persons: outcomes and predictors. J Am Geriatr Soc. 1994; 42(8):809-815. PubMed

Sepulveda E, Franco JG.Delirium en la versión en español del DSM-5: ¿más confusión? Rev Psiquiatr Salud Ment. 2015; 8:242-243. DOI 10.1016/j.rpsm.2015.05.002

Villalpando-Berumen JM, Pineda-Colorado AM, Palacios P, Reyes-Guerrero J, Villa AR, GutierrezRolncidence of delirium, risk factors, and long-term survival of elderly patients hospitalized in a medical specialty teach- ing hospital in Mexico City. Int Psychogeriatr. 2003; 15:325-336. PubMed

Wakefield BJ.Risk for acute confusion on hospital admission. Clin Nurs Res. 2002; 11:153-72. PubMed

Young J, Murthy L, Westby M, Akunne A, O’Mahony R.Diagnosis, prevention, and management of delirium: summary of NICE guidance BMJ. 2010; 341:c3704. DOI 10.1136/bmj.c3704

Young J, Murthy L, Westby M, Akunne A, O’Mahony R.Valoración del riesgo de delirium en pacientes mayores hospitalizados. Rev Esp Geriatr Gerontol. 2010; 45(5):285-290

DOI 10.1016/j.regg.2010.03.011 